\title{
Analysis of Starting Modes in FreQuency Controlled ASYNCHRONOUS ELECTRIC DRIVE FOR MINING PRODUCTION USING BUFFER POWER SUPPLIES.
}

\author{
Iosiff Breido \& Saifulin Ruslan
}
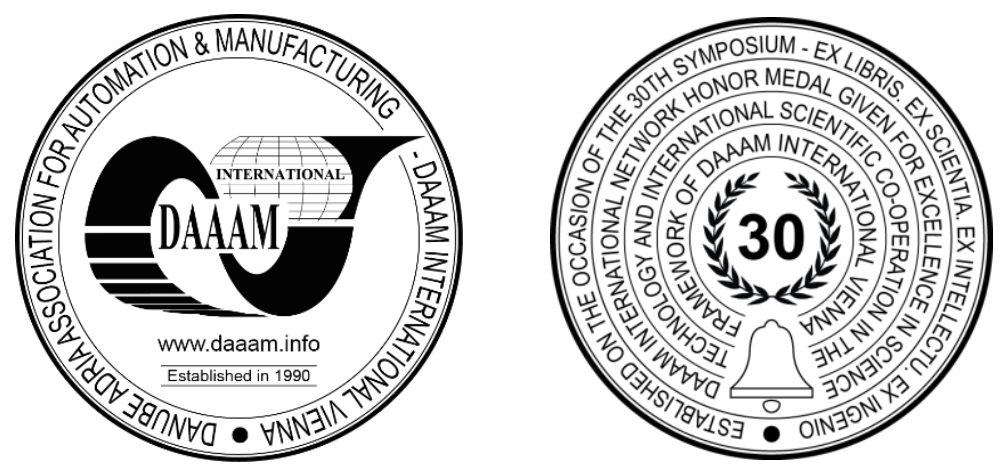

This Publication has to be referred as: Breido, I[osiff] \& Saifulin, R[uslan] (2020). Analysis of Starting Modes in Frequency Controlled Asynchronous Electric Drive for Mining Production Using Buffer Power, Proceedings of the 31st DAAAM International Symposium, pp.0118-0127, B. Katalinic (Ed.), Published by DAAAM International, ISBN 9783-902734-29-7, ISSN 1726-9679, Vienna, Austria

DOI: $10.2507 / 31$ st.daaam.proceedings.016

\begin{abstract}
The operation of an asynchronous electric drive for mining production in the regions of Central and Middle Asia is complicated by the fact that these facilities are located in remote from industrial networks areas. Because of this, the length of power lines can exceed the permissible length and there is a significant voltage drop in the network. In this paper the model of three-phase frequency-controlled asynchronous electric drive is developed using Simulink Matlab 2018 and converted into a two-phase model. It is proposed to compensate the voltage drop in the network for electric drive's starting modes with load by using Supercapacitors as a buffer power supply connected to the output of a DC link in a frequency converter. Simulation modeling was done at various supply voltages and various methods of connecting the Supercapacitor unit. It was found that such a principle of implementing an additional source of electrical energy allows to start asynchronous electric drives in areas remote from industrial networks without significantly affecting their static and dynamic characteristics.
\end{abstract}

Keywords: Electric drive; modeling; Matlab Simulink; Supercapacitor; transient response

\section{Introduction}

The study of a frequency-controlled asynchronous electric drive operation is a very urgent task. As known, power plants with an electric drive in the countries of Central and Middle Asia are often located in areas remote from industrial electrical networks. For such conditions the power of the source of electrical energy becomes comparable to the power of the electric drive due to losses in the extended network of power lines. [1] The length of a long power line exceeds the permissible length, which causes a voltage drop in industrial enterprises. Therefore, it becomes necessary to use additional autonomous power sources that create a combined power supply system for the electric drive, or to use various methods of starting the electric drive. These methods include powering from a diesel, gas turbine power plant, from an autonomous transformer, reactor start-up of electric drive, using of a synchronous generator. However, despite such a wide selection of methods for starting an electric drive in remote areas, each of these methods carries a number of disadvantages. 
So, as an example, in the papers of Anamika Dewangan, Virendra Sharm «SVPWM techniques used for controlling Three-Phase Induction Motor energized by renewable energy source» [2], Shevyrev Yu., Morgachev D. «Analysis of electromagnetic compatibility between diesel engine power plant and main electric motor of drilling unit» [3] alternative energy sources are used, as well as diesel generator sets as buffer power supply, however, this type of supply of electrical energy is not efficient as in the case of solar panels, as well as environmentally unfriendly and inconvenient as in the case of a diesel generator.

In addition, it is proposed to improve the power transmission networks directly by using filter compensating devices - Abramov B.I., Derzhavin D.A., Churikov D., Novoselov Yu. B., Suslov M.A., Shevyrev Yu.V. «Instrumental studies of the electrical energy quality for oil field under conditions of widespread application of frequency-regulated electrical drives» [4], but this method is also not rational due to the complexity of the idea's implementation, which consists in changing the structure of existing power transmission line systems.

Therefore, to solve this problem, it is proposed to use Supercapacitors as an autonomous buffer source of electrical energy. Supercapacitor (Ultracapacitor) - electrochemical device that can store a significant amount of energy. It consists of two plates - positively and negatively charged, which are placed in an electrolyte. During the charging of the Supercapacitor, ions from the electrolyte accumulate on the walls of this device. The key difference between a Supercapacitor and a battery is the absence of chemical reactions, which allows them to be charged and discharged many times faster, and they are also not subject to chemical wear, which allows them to be used in hundreds of thousands of charge and discharge cycles. These properties are excellent for an electric drive. The greatest load on the network occurs during the start of the electric motor, just at this moment subsidence occurs and there is a need for a buffer source of electricity that can instantly give out a certain amount of electrical energy.

Using of Supercapacitors as buffer energy sources is considered in papers of Belodedov A. «Model design of a controlled DC drive with a supercapacitor unit» [5], Girts Stana, Viesturs Brazis, Peteris Apse-Apsitis «Simulation of Induction Traction Drive with Supercapacitor energy storage system test bench» [6], Artan Ndokaj, A. Di Napoli, Giovanni Pede, Manlio Pasquali «Regulation strategy of an Ultracapacitor storage model for a gantry crane» [7], however, there are a number of issues in these works that were not considered. In all of these cases, only the static characteristics of the electric drive are evaluated, the dynamic operation of the drive is not considered.

Also, the main emphasis in these works is placed on the recuperation mode of operation, without affecting the electric drive start in case of a network drawdown and not evaluating the electric drive in dynamics. The drive models are taken from the Matlab Simulink standard library, which is designed with large assumptions and does not match real analogs used in the industry. In addition, in paper of Malligunta Kiran Kumar, Dhanekula Vallisa Datta, T Vijay Muni «Performance enhancement of Asynchronous Machine with Super Capacitor» [8] the block of Supercapacitors is connected via the G-E system, without using a frequency converter, which reduces its efficiency.

Thus, the study of starting modes in frequency controlled asynchronous electric drive for mining production with additional power supply from buffer sources is an urgent scientific and technical. Using of Supercapacitors in the DC link current of the frequency converter as a power source of the buffer, as well as its accumulation in the regenerative braking mode will allow solving the issues of power plants' power supply in areas remote from industrial networks, and will also save electrical energy by storing it during recuperation.

The work uses a method for modeling an asynchronous electric drive based on the Matlab Simulink software package, as well as its mathematical description using the basic laws of physics and Laplace operators, an analysis of the results obtained is also given.

\section{Wiring diagram of a Supercapacitor with an asynchronous electric drive}

In the process of developing the model, the method of direct connection of the Supercapacitor to the DC link in the frequency converter was used. In the future, it is planned to use a bi-directional DC-DC converter circuit to fully use the energy of the Supercapacitor. Wiring is shown in Figure 1.

Figure 1 also shows the control circuit of the Supercapacitor unit. The rectified value of the voltage is measured and compared with a predetermined. If, as a result of comparison, the rectified voltage is greater than or equal to the specified, then the key system is closed directly to the inverter subsystem, bypassing the Supercapacitor unit, and if the value of the rectified voltage of the industrial network turns out to be insufficient, then the key system connects the Supercapacitor unit in series with the DC link of the frequency converter, resulting in a buffer source of electrical energy. 


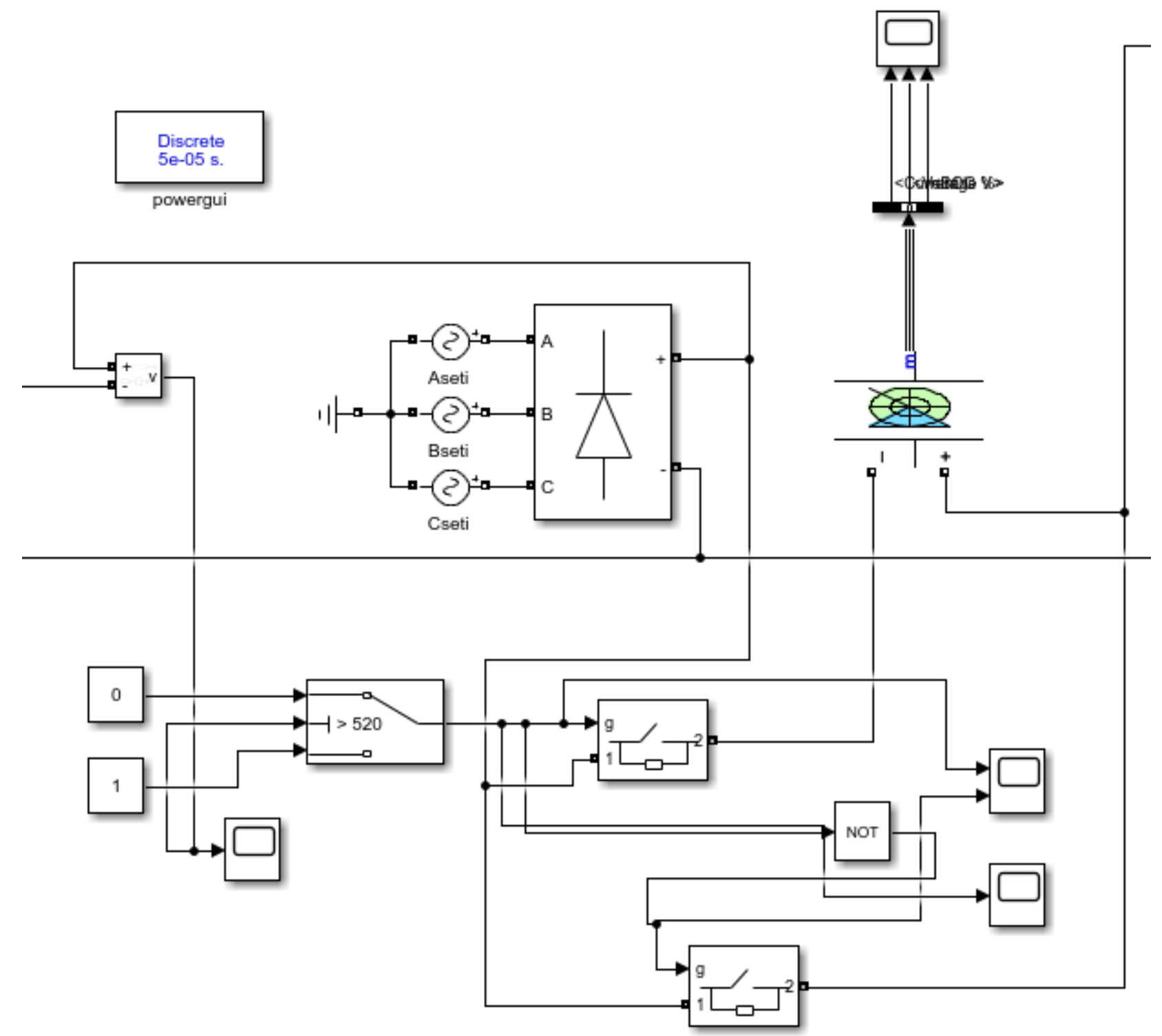

Fig. 1. Supercapacitor connection diagram

\section{Creation of a frequency-controlled asynchronous electric motor model with a block of Supercapacitors}

In this paper, a diagram of an AIR160S4 asynchronous electric motor with the following parameters presented in Table 1 is considered.

\begin{tabular}{|c|c|c|c|c|c|c|c|c|c|}
\hline $\begin{array}{l}\text { Electric } \\
\text { drive }\end{array}$ & $\begin{array}{l}\text { Power, } \\
\text { kW }\end{array}$ & $\begin{array}{c}\text { Speed, } \\
\text { rpm }\end{array}$ & Voltage, V & $\begin{array}{c}\text { Efficiency, } \\
\%\end{array}$ & $\begin{array}{l}\text { Power } \\
\text { factor }\end{array}$ & $I_{s} / I_{n}$ & $T_{s} / T_{n}$ & $T_{\max } / T_{n}$ & $\begin{array}{c}\text { Moment } \\
\text { inertia, } \\
\mathbf{k g ~ m}^{2}\end{array}$ \\
\hline АИР160S4 & 15 & 1450 & 400 & 89,5 & 0,86 & 7,7 & 2,2 & 2,6 & 0,075 \\
\hline
\end{tabular}

Table 1. AIR160S4 parameters

The model uses an asynchronous electric drive with scalar control via a frequency converter. The model is powered by a three-phase voltage shown in Figure 2. 


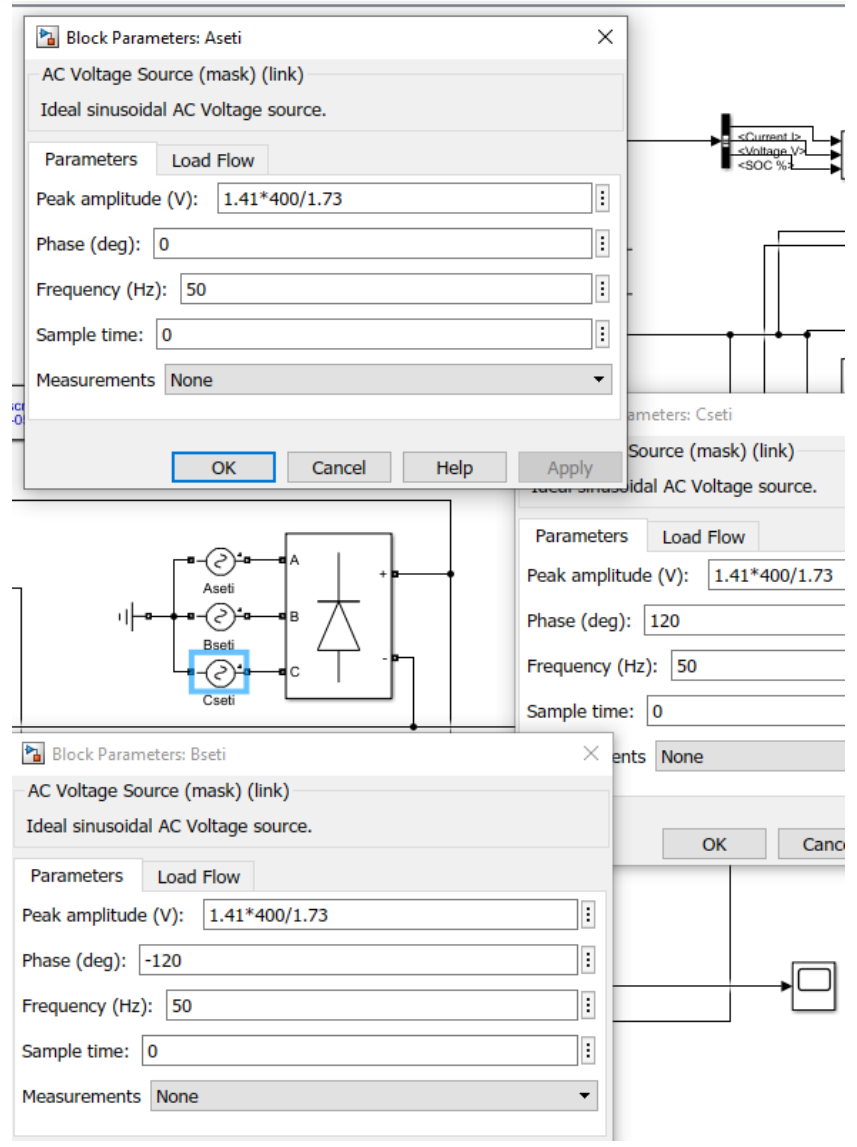

Fig. 2. System power model

The frequency converter consists of a diode bridge, a Supercapacitor and a system of switches, shown in Figure 1, as well as subsystems of inverters based on MOSFET transistors and their control signals $\mathrm{AH}, \mathrm{BH}, \mathrm{CH}, \mathrm{AL}, \mathrm{BL}, \mathrm{CL}$ implemented through the PWM generator shown in Figures 3 and 4.

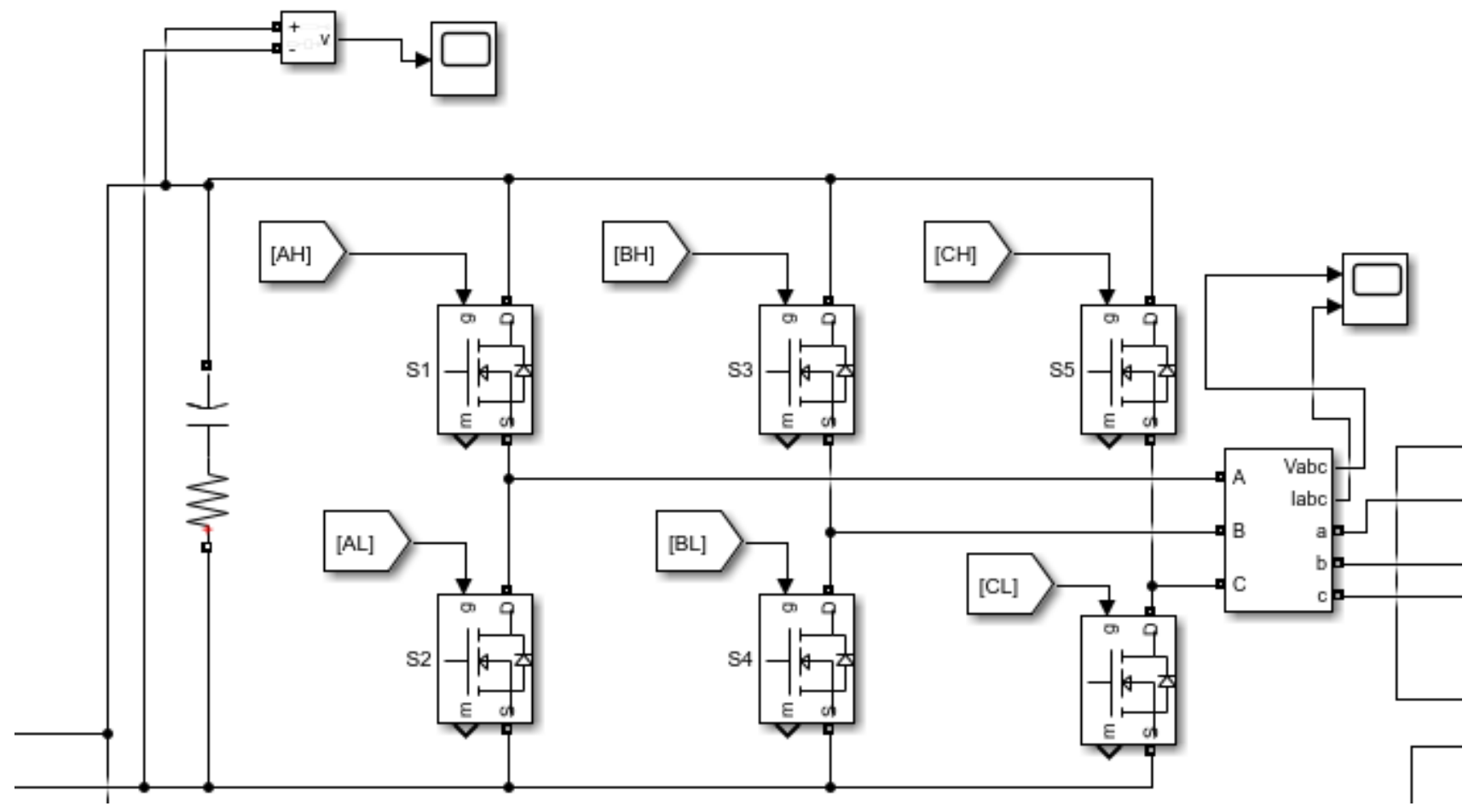

Fig. 3. Subsystem of inverters based on MOSFET transistors 


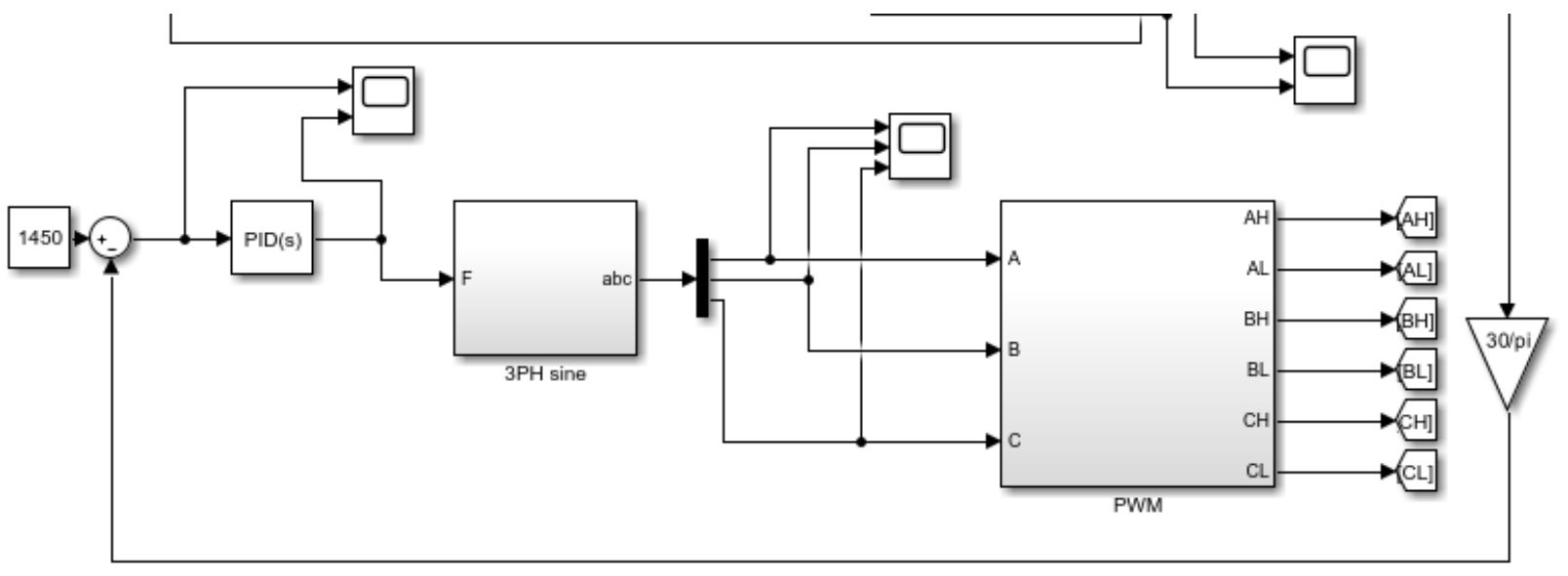

Fig. 4. PWM generator of control signals for transistors

In general, a three-phase voltage system is applied to the stator winding, offset by 120 degrees relative to each other. And the total voltage vector will be equal to the sum of the voltage vectors of each of the phases (1).

$$
\overrightarrow{U_{\Sigma}}=U_{m} \sin \omega t+U_{m} \sin \left(\omega t-\frac{2 \pi}{3}\right)+U_{m} \sin \left(\omega t+\frac{2 \pi}{3}\right)
$$

The projections of the vector $\overrightarrow{U_{\Sigma}}$ on the phase axes determine the instantaneous voltages on each of the phases A, B, C. All equations of current and flux linkage, which describe the operation of an AC motor, can be described in the same way.

However, phase converters $3 / 2$ are often used when designing real AC motor systems. A $3 / 2$ converter from a threephase voltage system A, B, C creates a two-phase $\alpha, \beta$. The transition to a new two-phase system allows to consider the spatial stress vector in a rectangular coordinate system. This change of variables is used in the mathematical description of electrical machines, and in particular, to simplify the solution and writing of the differential equations of the stator and rotor (2). [9]

$$
\left\{\begin{array}{c}
U_{\alpha}=\frac{2\left[U_{\mathrm{A}}-\frac{U_{\mathrm{B}}+U_{\mathrm{C}}}{2}\right]}{3} \\
U_{\beta}=\left(U_{\mathrm{B}}-U_{C}\right) / \sqrt{3}
\end{array}\right.
$$

After receiving the frequency-converted supply signal from the output of the frequency regulator, the voltage is converted from three-phase sinusoidal to two-phase to create a model of an asynchronous AC motor in a rotating coordinate system - Figure 5. As a result, the phase voltage $U_{A}, U_{B}, U_{C}$ is converted into $U_{X}, U_{Y}$.

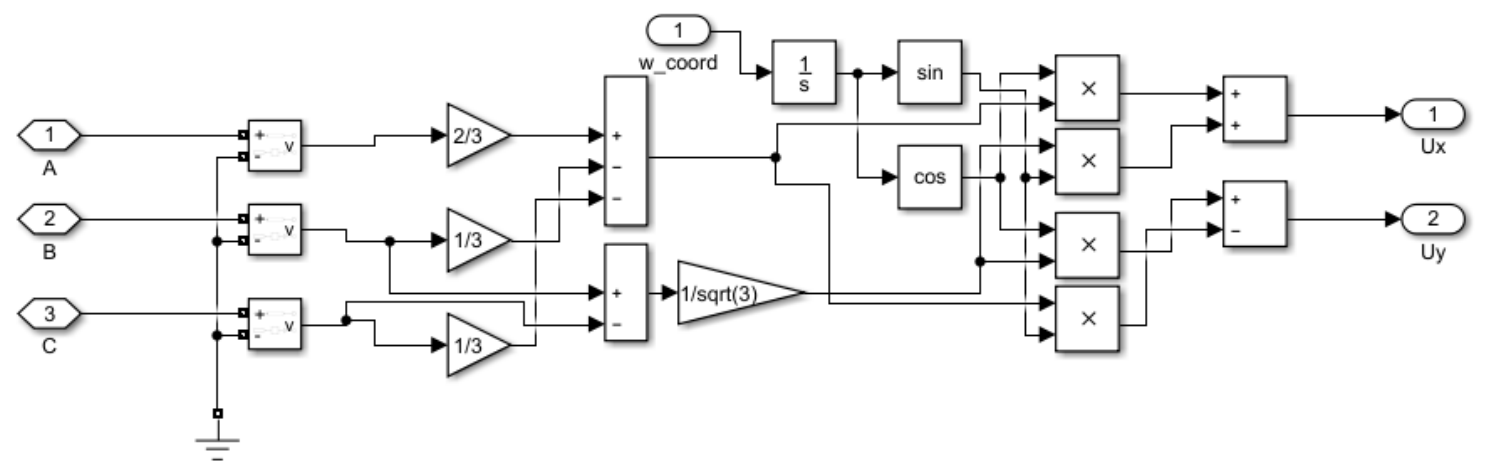

Fig. 5. Conversion of supply voltage

The resulting two-phase voltage is used to power an asynchronous AC motor model in a rotating coordinate system.

The system of equations for describing an asynchronous AC machine is based on four laws - Kirchhoff's second law, Ampere's law, Newton's second law and Lenz's left-hand rule. 


$$
\left\{\begin{array}{c}
\vec{U}_{S}=r_{S} \overrightarrow{l_{S}}+\frac{d \overrightarrow{\Psi_{S}}}{d t}+j \alpha_{k} \overrightarrow{\Psi_{S}} \\
\vec{U}_{R}=r_{R} \overrightarrow{\imath_{R}}+\frac{d \overrightarrow{\Psi_{R}}}{d t}+j\left(\alpha_{k}-p \vartheta_{m}\right) \overrightarrow{\Psi_{R}} \\
\overrightarrow{\Psi_{S}}=x_{S} \overrightarrow{\iota_{S}}+x_{m} \overrightarrow{\iota_{R}} \\
\overrightarrow{\Psi_{R}}=x_{R} \overrightarrow{\iota_{R}}+x_{m} \overrightarrow{\iota_{S}} \\
m=k \operatorname{Mod}\left(\overrightarrow{\Psi_{l}} x \overrightarrow{u_{k}}\right) \\
\overrightarrow{T_{m}} \frac{d \vartheta_{m}}{d t}=m-m_{c}
\end{array}\right.
$$

Based on the (3), a model of an asynchronous motor was calculated (formulas 4-32) and developed, this model is shown in Figure 6.

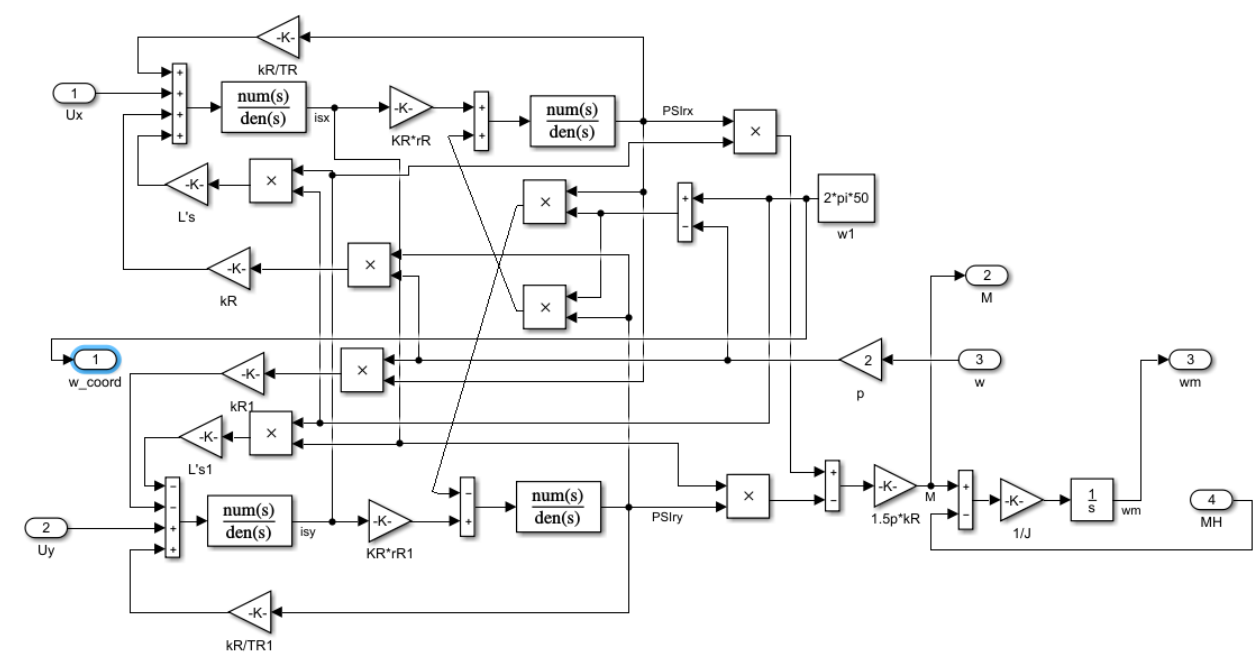

Fig. 6. Model of an asynchronous AC motor

$\vec{U}_{S}=r_{S} \overrightarrow{l_{S}}+\frac{d x_{S} \overrightarrow{l_{S}}+x_{m} \overrightarrow{l_{R}}}{d t}+j \alpha_{k}\left(x_{S} \overrightarrow{l_{S}}+x_{m} \overrightarrow{l_{R}}\right)$

$\vec{U}_{R}=0=r_{R} \overrightarrow{l_{R}}+\frac{d\left(x_{R} \overrightarrow{l_{R}}+x_{m} \overrightarrow{\underline{s}}\right)}{d t}+j\left(\alpha_{k}-p \vartheta_{m}\right)\left(x_{R} \overrightarrow{l_{R}}+x_{m} \overrightarrow{l_{S}}\right)$

$\frac{d \overrightarrow{l_{R}}}{d t}=-\frac{r_{R} \overrightarrow{l_{R}}}{x_{R}}-\frac{x_{m} d \overrightarrow{l_{S}}}{x_{R} d t}-j \alpha_{k} \overrightarrow{l_{R}}-j \alpha_{k} \frac{x_{m}}{x_{R}} \overrightarrow{l_{S}}+j p \vartheta_{m} \frac{\overrightarrow{\Psi_{R}}}{x_{R}}$

$\overrightarrow{\imath_{R}}=\frac{\overrightarrow{\Psi_{R}}-x_{m} \overrightarrow{l_{S}}}{x_{R}}$

$x_{m} \frac{d \overrightarrow{l_{R}}}{d t}=-\frac{r_{R} x_{m} \overrightarrow{\psi_{R}}}{x_{R}^{2}}+\frac{x_{m}^{2} \overrightarrow{\iota_{S}}}{x_{R}^{2}}-\frac{x_{m}^{2} d \overrightarrow{l_{S}}}{x_{R} d t}-j \alpha_{k} \overrightarrow{l_{R}}-j \alpha_{k} \frac{x_{m} \overrightarrow{\psi_{R}}}{x_{R}}+j p \vartheta_{m} \frac{x_{m} \overrightarrow{\psi_{R}}}{x_{R}}$

$\vec{U}_{S}=r_{S} \overrightarrow{l_{S}}+\frac{x_{S} d \overrightarrow{l_{S}}}{d t}-\frac{r_{R} x_{m} \overrightarrow{\Psi_{R}}}{x_{R}^{2}}+\frac{r_{R} x_{m}^{2} \overrightarrow{l_{S}}}{x_{R}^{2}}-\frac{x_{m}^{2} d \overrightarrow{l_{S}}}{x_{R} d t}+j \alpha_{k} x_{S} \overrightarrow{l_{S}}-\frac{j \alpha_{k} x_{m}^{2} \overrightarrow{l_{S}}}{x_{R}}+j p \vartheta_{m} \frac{x_{m} \overrightarrow{\Psi_{R}}}{x_{R}}$

$k_{R}=\frac{x_{m}}{x_{R}} ; r=r_{S}+k_{r}^{2} r_{R} ; x_{S}^{\prime}=x_{S}-\frac{x_{m}^{2}}{x_{R}} ; T_{R}=\frac{x_{R}}{r_{R}}$

$\overrightarrow{U_{S}}=r \overrightarrow{l_{S}}+\frac{x_{S}^{\prime} d \overrightarrow{l_{S}}}{d t}+j \alpha_{k} x_{S}^{\prime} \overrightarrow{l_{S}}-\frac{k_{R}}{T_{R}} \overrightarrow{\Psi_{R}}+j p \vartheta_{m} k_{R} \overrightarrow{\Psi_{R}}$

$\vec{U}_{R}=0=\frac{1}{T_{R}} \overrightarrow{\Psi_{R}}+\frac{d \overrightarrow{\Psi_{R}}}{d t}-k_{R} r_{R} \overrightarrow{l_{S}}+j\left(\alpha_{k}-p \vartheta_{m}\right) \overrightarrow{\Psi_{R}}$ 
$m=\overrightarrow{\Psi_{S \alpha}} \overrightarrow{l_{S \beta}}-\overrightarrow{\Psi_{S \beta}} \overrightarrow{l_{S \alpha}}$

$\overrightarrow{\Psi_{S}}=x_{S}^{\prime} \overrightarrow{l_{S}}+k_{R} \overrightarrow{\Psi_{R}}$

$\overrightarrow{\Psi_{S \alpha}}=x_{S}^{\prime} \overrightarrow{{ }_{S \alpha}}+k_{R} \overrightarrow{\Psi_{R \alpha}}$

$\overrightarrow{\Psi_{S \beta}}=x_{S}^{\prime} \overrightarrow{l_{S \beta}}+k_{R} \overrightarrow{\Psi_{R \beta}}$

$m=k_{R}\left(\overrightarrow{\Psi_{R \alpha} \overrightarrow{l_{S \beta}}}-\overrightarrow{\Psi_{R \beta}} \overrightarrow{l_{S \alpha}}\right)$

$\vec{U}_{S}=U_{S x}+j U_{S y} ; \vec{\imath}_{S}=i_{S x}+j i_{S y} ; \vec{\Psi}_{R}=\Psi_{R x}+j \Psi_{R y}$

$\left\{\begin{array}{c}\vec{U}_{S x}=r \overrightarrow{l_{S x}}+\frac{x_{s x}^{\prime} d \overrightarrow{l_{S x}}}{d t}-\alpha_{k} x_{S}^{\prime} \overrightarrow{l_{S y}}-\frac{k_{R}}{T_{R}} \overrightarrow{\Psi_{R x}}-p \vartheta_{m} k_{R} \overrightarrow{\Psi_{R y}} \\ \vec{U}_{S y}=r \overrightarrow{l_{S y}}+\frac{x_{s x}^{\prime} d \overrightarrow{l_{S y}}}{d t}-\alpha_{k} x_{S}^{\prime} \overrightarrow{l_{S x}}-\frac{k_{R}}{T_{R}} \overrightarrow{\Psi_{R y}}+p \vartheta_{m} k_{R} \overrightarrow{\Psi_{R x}} \\ \vec{U}_{R x}=0=\frac{1}{T_{R}} \overrightarrow{\Psi_{R x}}+\frac{d \overrightarrow{\Psi_{R x}}}{d t}-k_{R} r_{R} \overrightarrow{l_{S x}}-\left(\alpha_{k}-p \vartheta_{m}\right) \overrightarrow{\Psi_{R y}} \\ \vec{U}_{R y}=0=\frac{1}{T_{R}} \overrightarrow{\Psi_{R y}}+\frac{d \overrightarrow{\Psi_{R y}}}{d t}-k_{R} r_{R} \overrightarrow{l_{S y}}+\left(\alpha_{k}-p \vartheta_{m}\right) \overrightarrow{\Psi_{R x}} \\ m=k_{R}\left(\overrightarrow{\Psi_{R x}} \overrightarrow{l_{S y}}-\overrightarrow{\Psi_{R y}} \overrightarrow{l_{S x}}\right) \\ \overrightarrow{T_{m}} \frac{d \vartheta_{m}}{d t}=m-m_{c}\end{array}\right.$

$T_{S}^{\prime}=\frac{x_{S}^{\prime}}{r}$

$\left\{\begin{array}{c}\vec{U}_{S x}=r\left(1+T_{S}^{\prime} s\right) \overrightarrow{l_{S x}}-\alpha_{k} x_{S}^{\prime} \overrightarrow{l_{S y}}-\frac{k_{R}}{T_{R}} \overrightarrow{\Psi_{R x}}-p \vartheta_{m} k_{R} \overrightarrow{\Psi_{R y}} \\ \vec{U}_{S y}=r\left(1+T_{S}^{\prime} s\right) \overrightarrow{l_{S y}}+\alpha_{k} x_{S}^{\prime} \overrightarrow{l_{S x}}-\frac{k_{R}}{T_{R}} \overrightarrow{\Psi_{R y}}+p \vartheta_{m} k_{R} \overrightarrow{\Psi_{R x}} \\ \vec{U}_{R x}=0=\frac{1}{T_{R}}\left(1+T_{R} p\right) \overrightarrow{\Psi_{R x}}-k_{R} r_{R} \overrightarrow{l_{S x}}-\left(\alpha_{k}-p \vartheta_{m}\right) \overrightarrow{\Psi_{R y}} \\ \vec{U}_{R y}=0=\frac{1}{T_{R}}\left(1+T_{R} p\right) \overrightarrow{\Psi_{R y}}-k_{R} r_{R} \overrightarrow{l_{S y}}+\left(\alpha_{k}-p \vartheta_{m}\right) \overrightarrow{\Psi_{R x}} \\ m=k_{R}\left(\overrightarrow{\Psi_{R x}} \overrightarrow{l_{S y}}-\overrightarrow{\Psi_{R y}} \overrightarrow{l_{S x}}\right) \\ \overrightarrow{T_{m} s \vartheta_{m}}=m-m_{c}\end{array}\right.$

$\overrightarrow{l_{S x}}=\left(\vec{U}_{S x}+\alpha_{k} x_{S}^{\prime} \overrightarrow{l_{S y}}+\frac{k_{R}}{T_{R}} \overrightarrow{\Psi_{R x}}-p \vartheta_{m} k_{R} \overrightarrow{\Psi_{R y}}\right) \frac{1}{r\left(1+T_{S}^{\prime} p\right)}$

$\overrightarrow{l_{S y}}=\left(\vec{U}_{S y}-\alpha_{k} x_{S}^{\prime} \overrightarrow{l_{S x}}+\frac{k_{R}}{T_{R}} \overrightarrow{\Psi_{R y}}-p \vartheta_{m} k_{R} \overrightarrow{\Psi_{R x}}\right) \frac{1}{r\left(1+T_{S}^{\prime} p\right)}$

$\overrightarrow{\Psi_{R x}}=\left(k_{R} r_{R} \overrightarrow{l_{S x}}+\left(\alpha_{k}-p \vartheta_{m}\right) \overrightarrow{\Psi_{R y}} \frac{T_{R}}{1+T_{R} p}\right.$

$\vartheta_{m}=\frac{1}{\overrightarrow{T_{m} p}}\left(m-m_{c}\right)$ 
$\left\{\begin{array}{c}\vec{U}_{A}=U_{m} \sin (\omega t) \\ \vec{U}_{B}=U_{m} \sin \left(\omega t-\frac{2 \pi}{3}\right) \\ \vec{U}_{C}=U_{m} \sin \left(\omega t+\frac{2 \pi}{3}\right)\end{array}\right.$

$a=e^{j \frac{2 \pi}{3}}=-\frac{1}{2}+j \frac{\sqrt{3}}{2}$

$a^{2}=e^{j \frac{4 \pi}{3}}=-\frac{1}{2}-j \frac{\sqrt{3}}{2}$

$\vec{U}_{S}=\frac{2}{3}\left(\vec{U}_{A}+a \vec{U}_{B}+a^{2} \vec{U}_{C}\right)$

$\vec{U}_{S}=\frac{2}{3}\left[U_{m} \sin (\omega t)+\left(-\frac{1}{2}+j \frac{\sqrt{3}}{2}\right) U_{m} \sin \left(\omega t-\frac{2 \pi}{3}\right)+\left(-\frac{1}{2}-j \frac{\sqrt{3}}{2}\right) U_{m} \sin \left(\omega t+\frac{2 \pi}{3}\right)\right]$

$\sin \left(\omega t \pm \frac{2 \pi}{3}\right)=-\frac{1}{2} \sin (\omega t) \pm \frac{\sqrt{3}}{2} \cos (\omega t)$

$\vec{U}_{S}=U_{m} \sin (\omega t)-j U_{m} \cos (\omega t)$

\section{Simulation of starting an asynchronous motor with a Supercapacitor block}

After the model of the entire system was created, virtual experiments with the combined power supply began.

Experiment \# 1 - nominal mains supply - $400 \mathrm{~V}$, drive started under load at time $0.055 \mathrm{sec}$. Since the voltage is nominal, the Supercapacitor unit is disconnected from the supply system at this moment. Graphs of torque, speed, flux linkage and stator current are shown in Figure 7.

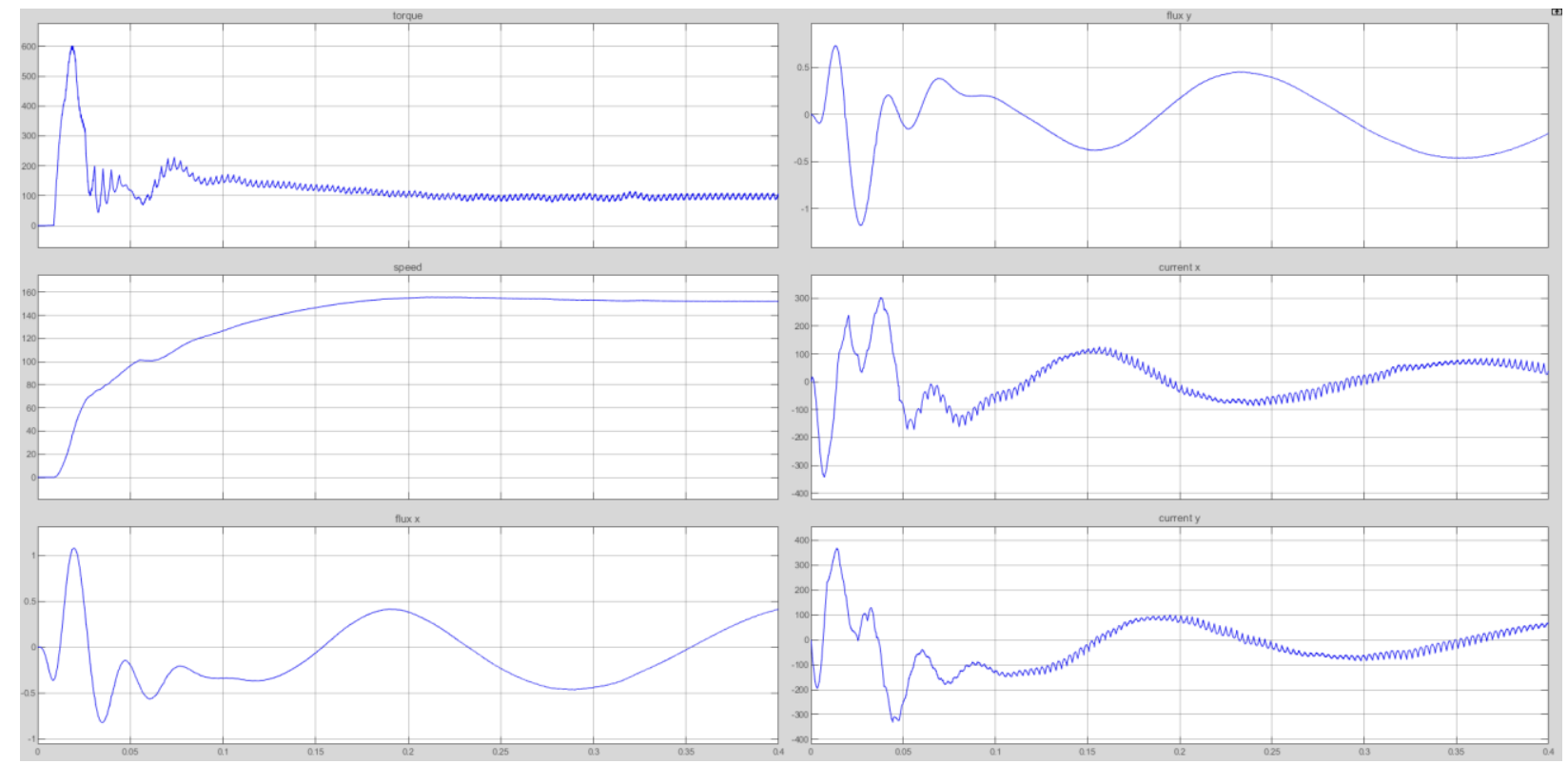

Fig. 7. Characteristics at $400 \mathrm{~V}$

As you can see from the graphs, the motor develops a rated torque of $97.5 \mathrm{~N}$, as well as a rated speed of $152 \mathrm{rad} / \mathrm{s}$. At the same time, at the time of $0.055 \mathrm{sec}$. there is a torque drawdown due to load connection. The process is monotonous, the overshoot is $1.97 \%$, the transient time is $0.2 \mathrm{sec}$.

Experiment \# 2 - the network drawdown is assumed to be $20 \%$, which will be $320 \mathrm{~V}$ of the supply voltage, the Supercapacitor unit is disabled in this experiment. The graphs of torque, speed, flux linkage and stator current are shown in Figure 8. 


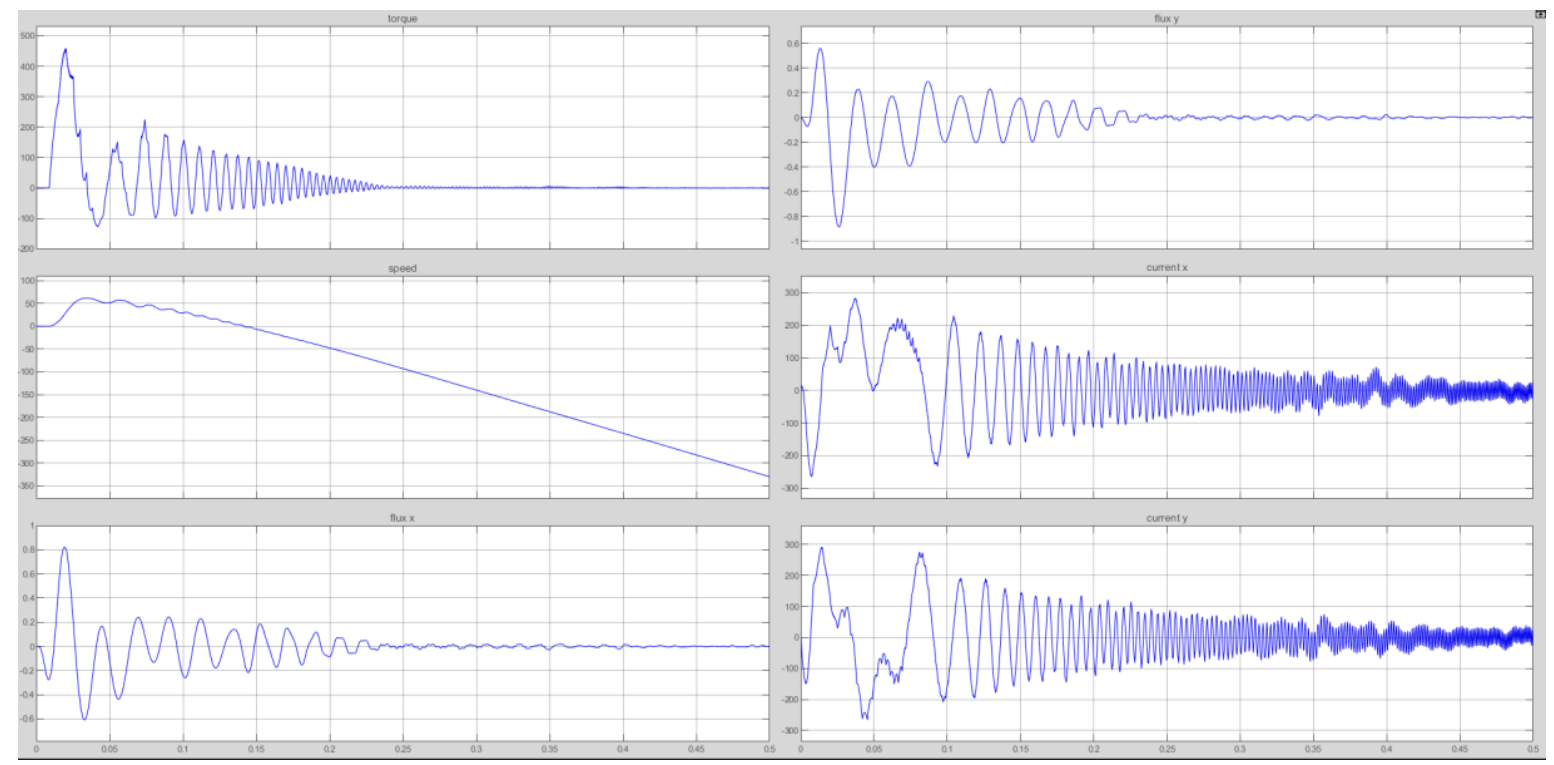

Fig. 8. Characteristics at $320 \mathrm{~V}$

At a given voltage of the supply network, when the load is connected, the motor cannot overcome the moment of resistance forces and overturns. The system becomes unstable.

Experiment \# 3 - the network drawdown is also assumed to be $20 \%$, the supply voltage is respectively equal to 320 $\mathrm{V}$, but at the same time the combined power supply system of the electric motor is activated. Additional voltage of the Supercapacitor block will be used. The graphs are shown in Figure 9.

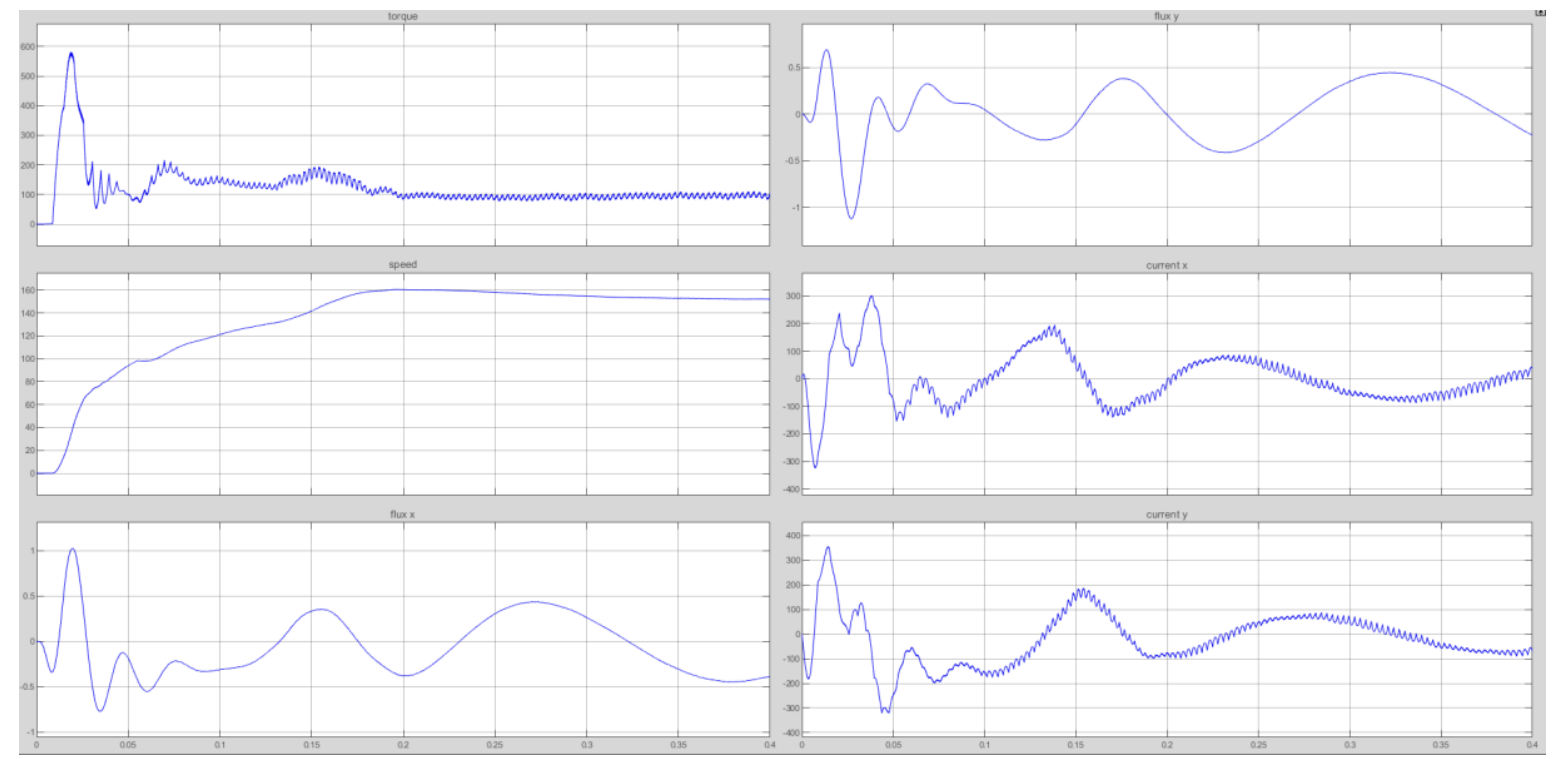

Fig. 9. Characteristics at $320 \mathrm{~V}$ and a Supercapacitor

These graphs show that, thanks to a block of Supercapacitors acting as buffer energy sources, motor at the moment of load connection at $0.055 \mathrm{sec}$. manage to reach nominal values. At the same time, the time of the transient process increased by $0.1 \mathrm{sec}$. and overshoot up to $5.26 \%$. However, these values are acceptable when assessing the quality of the transient. From a monotonous transient process has turned into aperiodic. However, it was precisely the use of a block of Supercapacitors as an additional source of electrical energy that made it possible to start an asynchronous motor with a drawdown of the supply network of $20 \%$.

Figure 10 shows the rectified mains voltage and the combined voltage of the network and the Supercapacitor unit at the output of the DC link in the frequency converter. The graphs show a slight effect of the Supercapacitor block on the output voltage from the DC link. The amount of oscillation is $10 \%$ and continues for 0.03 seconds. According to these data, it can be judged that the Supercapacitor unit does not affect the quality characteristics of the electric drive's supply voltage. 


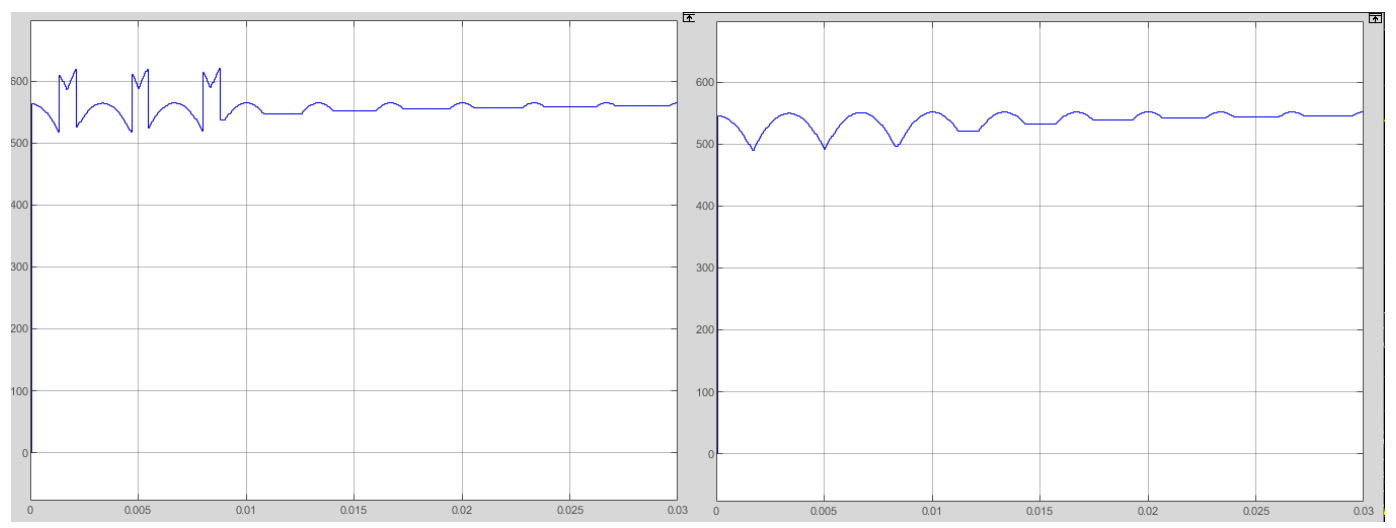

Fig. 10. Graph of rectified mains voltage and combined with a block of Supercapacitors

\section{Conclusion}

Problems arise in starting modes when power supply of the mining industry or drilling rigs electric drive located at a considerable distance from power sources. Unacceptable voltage drops may occur, resulting in reduced starting torque. The paper describes the development of a frequency-controlled electric drive of mining machines mathematical model for operation in conditions of remoteness from power supply sources.

With a voltage drop of more than $20 \%$, the implementation of the start-up mode under load is impossible, which is confirmed by simulation experiments in the paper. To solve this problem, it is proposed to use a buffer energy storage. The use of a Supercapacitor as a buffer source of electrical energy in the DC link in a frequency converter of an AC asynchronous motor when it is started in areas remote from industrial networks allows compensating voltage drops in the network, to ensure satisfactory starting conditions. Analysis of simulation experiments for starting an asynchronous motor showed a slight increase in the duration of the transient process, as well as a slight increase in system oscillation due to an increase in the overshoot value, but at the same time these indicators of the quality of the transient process remain satisfactory.

Thus, the use of Supercapacitors as buffer sources of electricity for starting an AC asynchronous motor from a source of comparable power is acceptable and expands the use of variable frequency asynchronous electric drives in the mining industry. The direction of further research is the analysis of transients at the moments of connecting and disconnecting Supercapacitors. An analysis of energy storage in the form of recuperation during operation will also be carried out. Development of a buffer source is planned.

\section{References}

[1] Breido I.; Kaverin V. \& Em G. (2018) The research of the adjustable electric drive of the direct current DAAAM International Scientific Book 2018 Vol. 17, p 211-226, ISSN 1726-9687

[2] Dewangan, Anamika \& Sharma, Virendra. (2017). SVPWM Techniques Used for Controlling Three-Phase Induction Motor Energized by Renewable Energy Source. 3297. 2320-3765. 10.15662/IJAREEIE.2017.0602017

[3] Shevyrev, Yu \& Morgachev, D.. (2015). Analysis of electromagnetic compatibility between diesel engine power plant and main electric motor of drilling unit. Gornyi Zhurnal. 2015. 10.17580/gzh.2015.01.11

[4] Abramov, B.I. \& Derzhavin, D.A. \& Churikov, D. \& Novoselov, Yu.B. \& Suslov, M.A. \& Shevyrev, Yu.V.. (2016). Instrumental studies of the electrical energy quality for oil field under conditions of widespread application of frequency-regulated electrical drives. 90-92

[5] Belodedov, A. Model design of a controlled DC drive with a supercapacitor unit [Electronic resource] / A. Belodedov, O. Lysenko // Dynamics of Systems, Mechanisms and Machines : conference proceeding, 15-17 November 2016 / Omsk State Technical University. - Omsk, 2016. - DOI: 10.1109/Dynamics.2016.7818978

[6] Stana, Girts \& Brazis, Viesturs \& Apse-Apsitis, Peteris. (2015). Simulation of Induction Traction Drive with Supercapacitor Energy Storage System Test Bench. Electrical, Control and Communication Engineering. 9. 10.1515/ecce-2015-0007

[7] Ndokaj, Artan \& Napoli, A. \& Pede, Giovanni \& Pasquali, Manlio. (2013). Regulation strategy of an Ultracapacitor storage model for a gantry crane. IECON Proceedings (Industrial Electronics Conference). 12091216. 10.1109/IECON.2013.6699305

[8] Kumar, M.K. \& Datta, D.V. \& Muni, Vijay. (2019). Performance enhancement of asynchronous machine with super capacitor. International Journal of Engineering and Advanced Technology. 8. 1208-1210

[9] Yu. N. Dementyev; V. B. Terekhin;I. G. Odnokopylov; V. M. Rulevskiy. Computer simulation of electromechanical AC-DC systemsin MATLAB Simulink [in Russian] / Study guide. Tomsk Polytechnic University. - Tomsk:Tomsk Polytechnic University Publishing, 2018., pp. 43-47, ISBN 978-5-4387-0819-3 